\title{
INVERSE IDENTIFICATION OF THE BENDING STIFFNESS OF A BRAIDED POLYETHYLENE TWINE SUBJECT TO LARGE DEFORMATION: APPLICATION TO THE IDENTIFICATION OF THE MESH OPENING RIGIDITY OF FISHING NETS.
}

\author{
B. Morvan $^{1-2}$, G. Bles ${ }^{2}$, N. Dumergue ${ }^{1}$, and D. Priour ${ }^{1}$ \\ ${ }^{1}$ IFREMER \\ Plouzane, France \\ e-mail: \{barthelemy.morvan, nicolas.dumergue, daniel.priour\}@ifremer.fr \\ ${ }^{2}$ ENSTA Bretagne \\ FRE CNRS 3744 \\ IRDL \\ F-29200 Brest, France \\ e-mail: guilhem.bles@ensta-bretagne.fr
}

Keywords: Numerical model, experiments, braided twine, mesh opening rigidity, large deformation.

\begin{abstract}
The evaluation of the mesh opening stiffness of fishing nets is an important issue in assessing the selectivity of trawls. It appeared that a larger bending rigidity of twines decreases the mesh opening and could reduce the escapement of fish. Nevertheless, netting structure is complex. A netting is made up of braided twines made of polyethylene or polyamide. These twines are tied with non-symmetrical knots. Thus, these assemblies develop contact-friction interactions. Moreover, the netting can be subject to large deformation. In this study, we investigate the responses of netting samples to different types of solicitations. Samples are loaded and unloaded with creep and relaxation stages, with different boundary conditions. Then, two models have been developed: an analytical model and a finite element model. The last one was used to assess, with an inverse identification algorithm, the bending stiffness of twines. In this paper, experimental results and a model for netting structures made up of braided twines are presented. During dry forming of a composite, for example, the matrix is not present or not active, and relative sliding can occur between constitutive fibres. So an accurate modelling of the mechanical behaviour of fibrous material is necessary. This study offers experimental data which could permit to improve current models of contact-friction interactions [4], to validate models for large deformation analysis of fibrous materials [1] on a new experimental case, then to improve the evaluation of the mesh opening stiffness of a fishing net.
\end{abstract}




\section{INTRODUCTION}

Twines used in the manufacture of netting materials for trawl codends tend to be stiffer. This increased stiffness affects the mesh opening in fishing gears, and thus the ability of fish to escape. The strong influence of the codend on trawl selectivity has been demonstrated [15]. Moreover, previous studies have shown how mesh rigidity to opening affects the mechanical behaviour, and thus the selectivity of codends ([5], [16]). However, the mesh opening stiffness is quite difficult to evaluate. In fact, it depends on its definition and on the theoretical model of the netting. Generally, to assess the mesh opening stiffness, we consider that a mesh side must have the same behaviour as a beam ([16], [9], [12], [13]). With this theoretical model, it appears that the bending rigidity EI of the beam represents the mesh opening stiffness. $\mathrm{O}^{\prime}$ Neill demonstrated how an increase in twine bending stiffness reduces the diameter of the codend and so the mesh opening. In addition, Herrmann [5] reported that an increase in twine bending stiffness could lead to a reduction in selectivity. Likewise, Moderhak [7] theoretically demonstrated how changes in mesh size and mesh opening stiffness can impact the shape of a codend and its selectivity.

As far as the authors know, the first method implemented so far for the assessment of this stiffness was presented by Sala [16]. The method uses a prototype on which netting panels are mounted and deformed. It incorporates four tension load cells and four stepping motors and it is designed so that all twines of the netting panel have the same deformation. The bending stiffness of the netting twine is estimated from the data of forces and deformation using the beam theory. However, the prototype uses a relatively expensive and high-tech device to ensure the uniform deformation of the netting panel.

Alternative methods exist: Priour and Cognard [14] assumed that the twine bending stiffness could be given by the stiffness of a simple cantilever beam whose deformation equals the observed deformation of a netting panel subject to out-of-plan bending. However, the method does not take into account the effect of knots and requires a sample of close mesh netting. De la Prada and Gonzalez [12] offered a simple uniaxial experimental set-up, which stretches a netting sample in the T-direction of the meshes while leaving free its deformation in the N-direction. Nevertheless, they assumed that the deformation is identical in all meshes of the sample. This assumption is probably too strong because the panel is held in the vertical position during the experiment, so forces and deformation supported by meshes at the top of the panel are higher than those supported at the bottom due to gravity.

The final aim of this study is to propose a simple methodology to assess the mesh opening stiffness of fishing nets. This methodology has to be based on a simple experimental plan, which does not require expensive devices, to be easily used in laboratories and in the fishing industry.

In this paper, we investigate the responses of netting samples to different types of solicitations; samples are loaded and unloaded with creep and relaxation stages, with two different boundary conditions. Then, two models have been developed: an analytical model and a finite element model. The last one allows the simulation of the netting without uniform deformation. These models were used to assess, with an inverse identification algorithm, the bending stiffness of mesh sides. In this paper, experimental results and models for netting structures made up of braided twines are presented. 


\section{MATERIAL AND EXPERIMENTAL METHOD}

\subsection{Netting}

The netting materials specifications (fibre type, twine construction) were selected according to the netting commonly used in trawl codends.

In this paper, we investigate a single twine netting made up of polyethylene (PE). This twine is made up of a core and a sheath (Fig. 1). The core and the sheath are composed of 20 and 64 fibres respectively. In the core, fibres are twisted, whereas in the sheath 16 threads made up of 4 fibres are braided. The linear density of the twine is $5590.410^{-6} \pm 10.610^{-6} \mathrm{~kg} \cdot \mathrm{m}^{-1}$, that is $5590.4 \pm 10.6$ tex. The diameter of the fibres, measured with a digital microscope, is 302.1 $\mu \mathrm{m}$. The diameter of the twine is $3.14 \pm 0.01 \mathrm{~mm}$. The pitch of the braided sheath, which is the longitudinal distance required for one revolution of a thread around the twine, is $26.12 \pm 0.62$ $m m$.
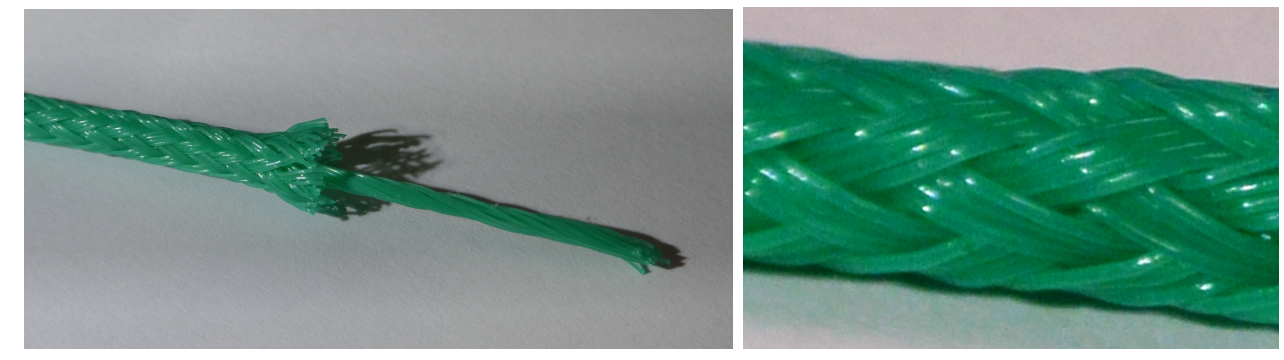

Figure 1: Left: single twine netting made up of polyethylene. Right: the braided sheath.
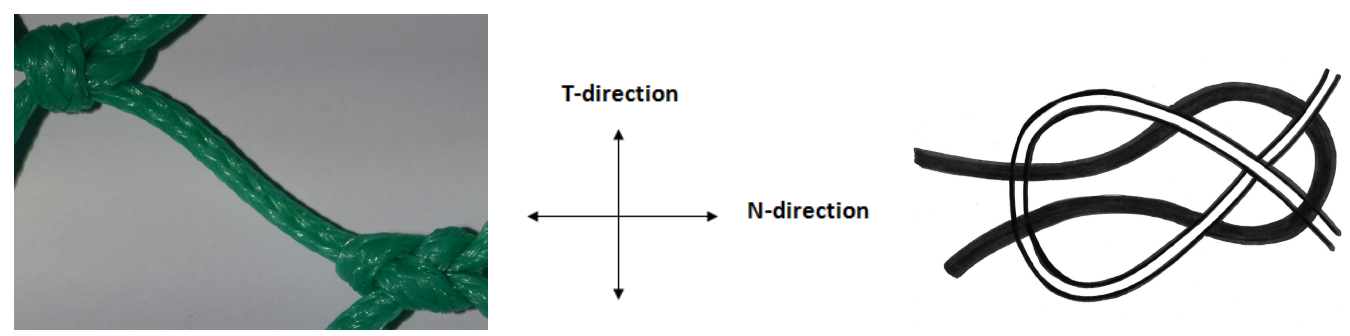

Figure 2: Left: mesh side in a single twine netting made up of polyethylene braided twines. Right: scheme of the knot.

Each 4x10 mesh netting panel was subject to a pretension step: each sample was loaded in tensile with $392.4 \mathrm{~kg}$ in the $\mathrm{N}$-direction during 30 minutes.

In order to define the input data of models, the sample characteristics are obtained:

- The mass $m$ of the netting panel is measured using electronic scale.

- The characteristic length of the netting mesh sides is obtained from the mean value of some mesh side lengths measured with a caliper. The length of the mesh side is $40 \mathrm{~mm}$.

- The diameter of the beam elements is calculated so that the mass of the model of the netting panel is equal to the mass of the real one. The material density of high-density polyethylene used for the calculation of the diameter is $950 \mathrm{~kg} \cdot \mathrm{m}^{-3}$. 
- The initial position of the netting panel (i.e. the position at rest) is derived from the length and the width of the panel at rest. The panel position at rest is performed by vibrating a horizontal plane where the netting panel is put down free of load, until a stable position is reached. Then, the dimensions (width $W_{\text {panel0 }}$, length $L_{\text {panel0 }}$ ) of the netting panel are measured and the mesh angle $\alpha_{0}$ (angle between a mesh side and the $\mathrm{N}$-direction) is derived with a simple cosinus equation using the characteristic length of the mesh sides (Fig. 3).

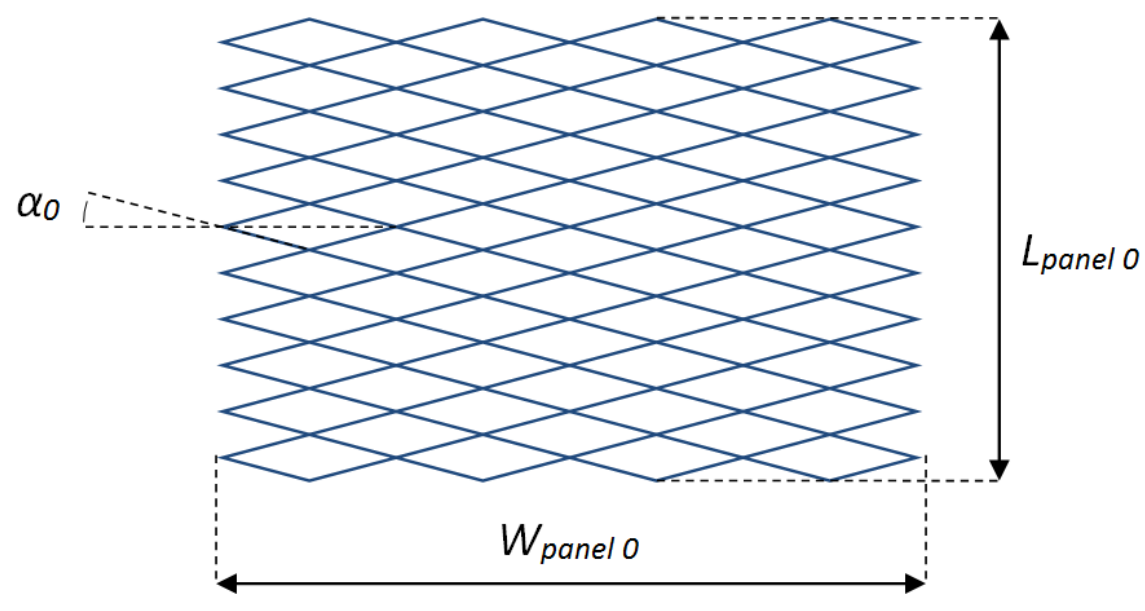

Figure 3: The dimensions $W_{\text {panel }_{0}}$ and $L_{\text {panel }_{0}}$ of the netting panel allow the mesh angle $\alpha_{0}$ at rest to be calculated.

For the study, it is convenient to define some parameters. First, the T-direction and the Ndirection in a mesh of the netting have been defined in [6]. The two directions are defined relatively to the knot orientation (Fig. 2 and 4 ).

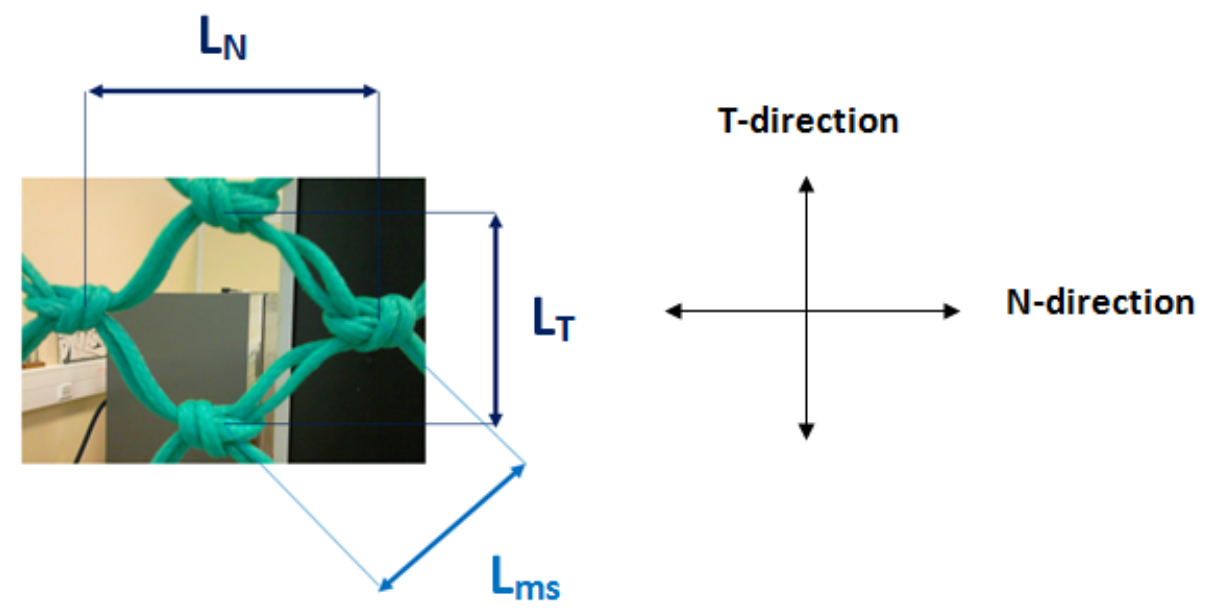

Figure 4: Definition of the N-direction and of the T-direction, and definition of the parameters $L_{N}, L_{T}$ and $L_{m s}$ in a diamond netting mesh. 
Then, we can define, in one mesh, the distances $L_{N}$ and $L_{T}$ between opposite knots, in the $\mathrm{N}$-direction and the T-direction respectively. $L_{m s}$ is the length of the mesh side at rest.

To work with dimensionless parameters which do not depend on the mesh side length, we introduce the openings $o_{N}$ and $o_{T}$ in the $\mathrm{N}$-direction and the T-direction respectively. The opening $o_{i}$, in the direction $i$, is the ratio of the distance $L_{i}$ by the mesh side length $L_{m s}$ (Eq. 1).

$$
o_{i}=\frac{L_{i}}{L_{m s}}
$$

\subsection{Evaluation of the axial stiffness}

Tensile tests have been performed on a LR5Kplus tensile testing machine to evaluate the axial stiffness of twines constituting the studied fishing nets. Because of the complex visco-elastoplastic behaviour of the polyethylene, it is not straightforward to evaluate the axial stiffness of the instantaneous elasticity. We proposed to assess this stiffness by measuring the moduli of short-time behaviour according to [2]. G. Bles [2] noted that the measurements after relaxations and creeps and those of the initial modulus were in agreement and provided a characteristic evolution of the modulus. In our study, the strain rate was $2.10^{-4} \mathrm{~s}^{-1}$ and the load at the beginning of the relaxation stages increased from 100 to $900 \mathrm{~N}$, by $100 \mathrm{~N}$. The duration of the eight relaxation stages was 15 minutes.

We calculated the moduli of short-time behaviour by calculating the slope at the beginning of each load step, just after a relaxation stage (Fig. 5). The evolution of the moduli with the logarithmic strain in the case of the studied braided twine is shown in figure 5 . For a small logarithmic strain $(<0.055)$, we will suppose that the modulus of short-time behaviour, which means the axial stiffness, is inferior to $14000 \mathrm{~N}$.
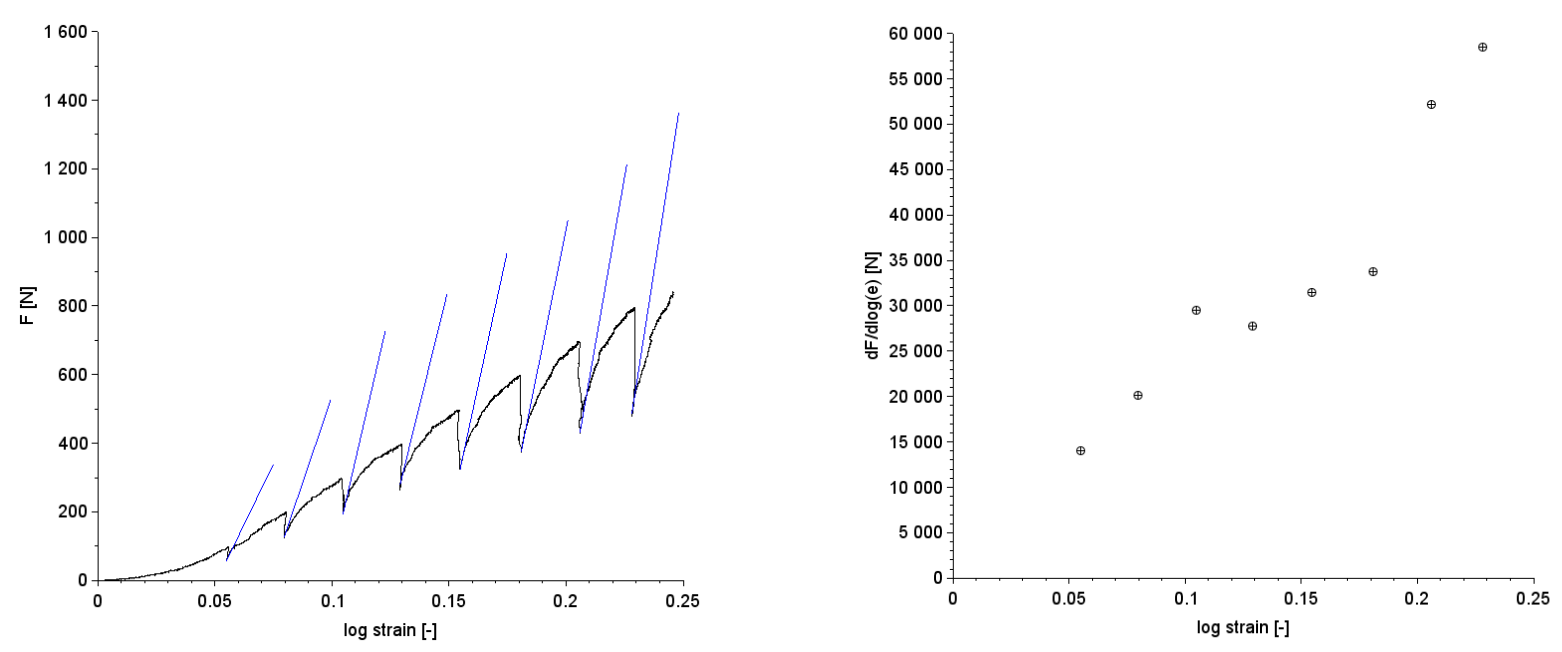

Figure 5: Left: the black line presents the load as a function of the logarithmic strain, and the blue segments indicate the slope (short-time modulus) just after each relaxation stage. Right: evolution of the short-time modulus, obtained with the slopes in the figure on the left.

\subsection{Experiments}

Two types of experiments were performed: a tensile test, on a classical testing machine, and a suspending test of the same type as [11]. 


\subsubsection{Tensile test}

In the first type, measurements were performed on a LR5Kplus tensile testing machine, with a $250 \mathrm{~N}$ load cell, of the company Lloyd instruments. The uniaxial tension tests were controlled by the jaw displacement. The relaxation stages were performed by blocking the jaw movement. A LASERSCAN 200 non-contacting extensometer (Lloyd instruments) allowed the measurement of the height of the mesh in the middle of the sample (Fig 6).

Concerning the load, we measured the sum of the effects of the displacement, the weight of the netting panel and the weight of the device which allowed the fixation of the sample. We defined the loads $F m_{N}$ and $F m_{T}$ as the loads applied on one mesh in the N-direction and the Tdirection respectively. So, knowing the load $F$ applied on the netting panel, the weight $P_{\text {panel }}$ of the sample and the weight $P_{\text {device }}$ of the device, we calculated the force by mesh $F m_{T}$ applied on the mesh in the middle of the netting sample:

$$
F m_{T}=\frac{F-\frac{P_{\text {panel }}}{2}-P_{\text {device }}}{4}
$$
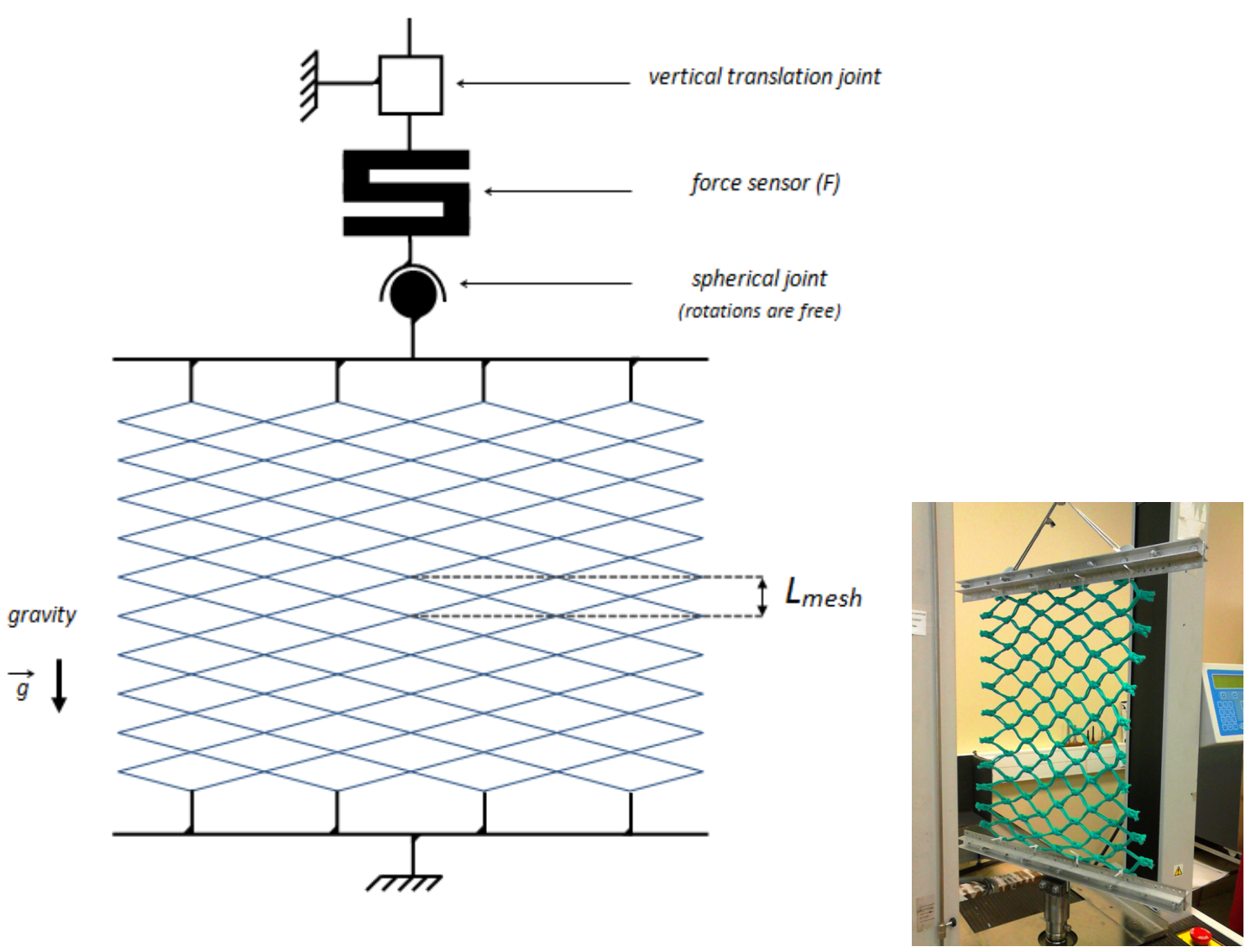

Figure 6: Plan (left) and photograph (right) of the experimental set up of the tensile test. 


\subsubsection{Suspending test}

In the second type of experiment, the panel was suspended from one of its ends and was subject to its own weight and to forces applied on the four nodes at the bottom (Fig. 7).

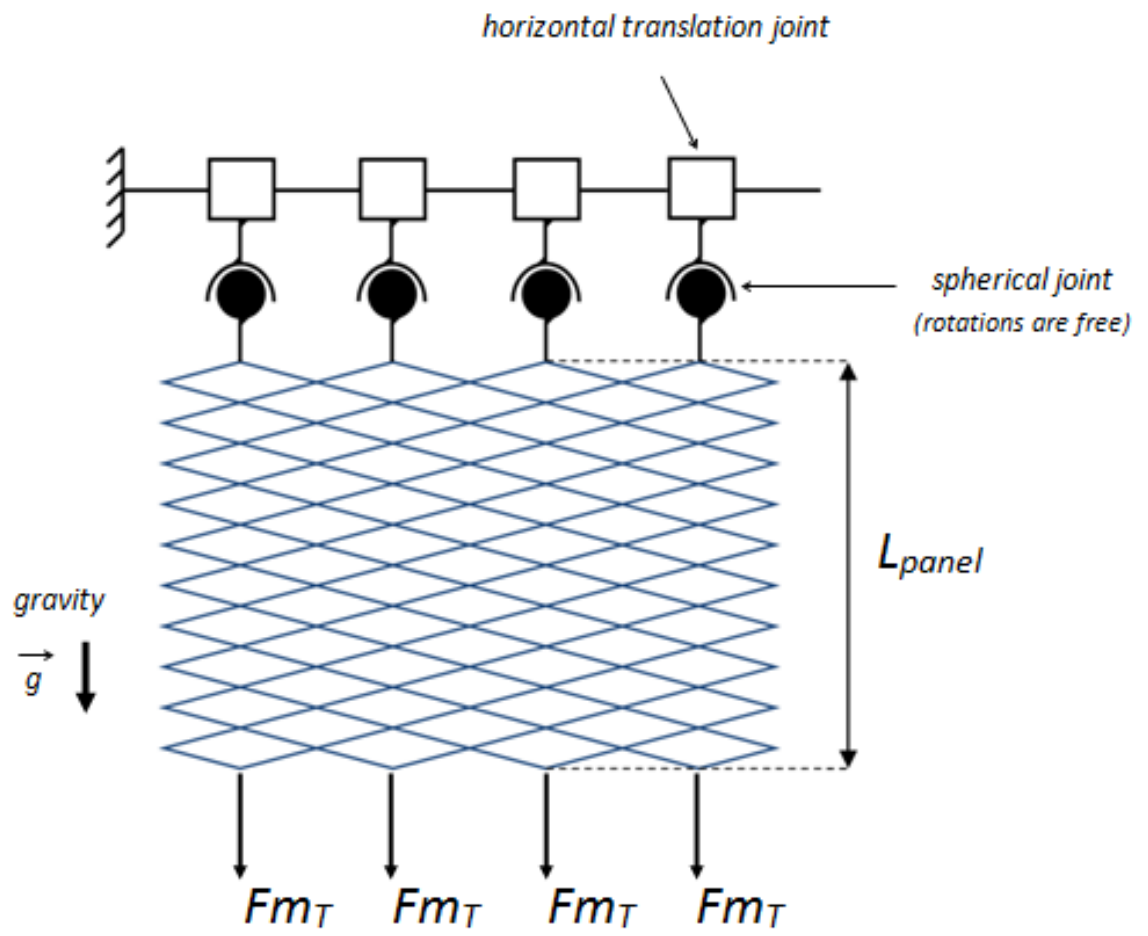

Figure 7: Experimental set up plan of the suspending test. A panel is suspended from one of its ends and is subject to its own weight and to forces applied on its bottom end.

Because of the possible variation in the results, 10 samples of $4 \times 10$ mesh netting panels were tested in the same conditions and loadings.

When a panel was suspended, the positions of all the nodes of the netting panel were measured. The use of a camera with a software designed and implemented in the laboratory allowed the recording of pictures with a chosen frequency. Note that the software allowed the application of optical corrections. Then, targets defined by the user on the first picture were identified in all the pictures. So the displacements of all these targets were measured during the recording. The height of the netting panel $L_{\text {panel }}$ is measured using the position of the uppermost knot and the position of the lowermost knot.

\section{EXPERIMENTAL RESULTS}

\subsection{Tensile test}

Results of a tensile test are shown on figures 8 and 9. A load and unload cycle interrupted by several relaxation stages was imposed. The controlled jaw displacement rate was $100 \mathrm{~mm} . \mathrm{min}^{-1}$. The duration of the 9 relaxation stages was 15 minutes.

Figure 10 presents the result of a cyclic tensile test on a double twine netting sample. 

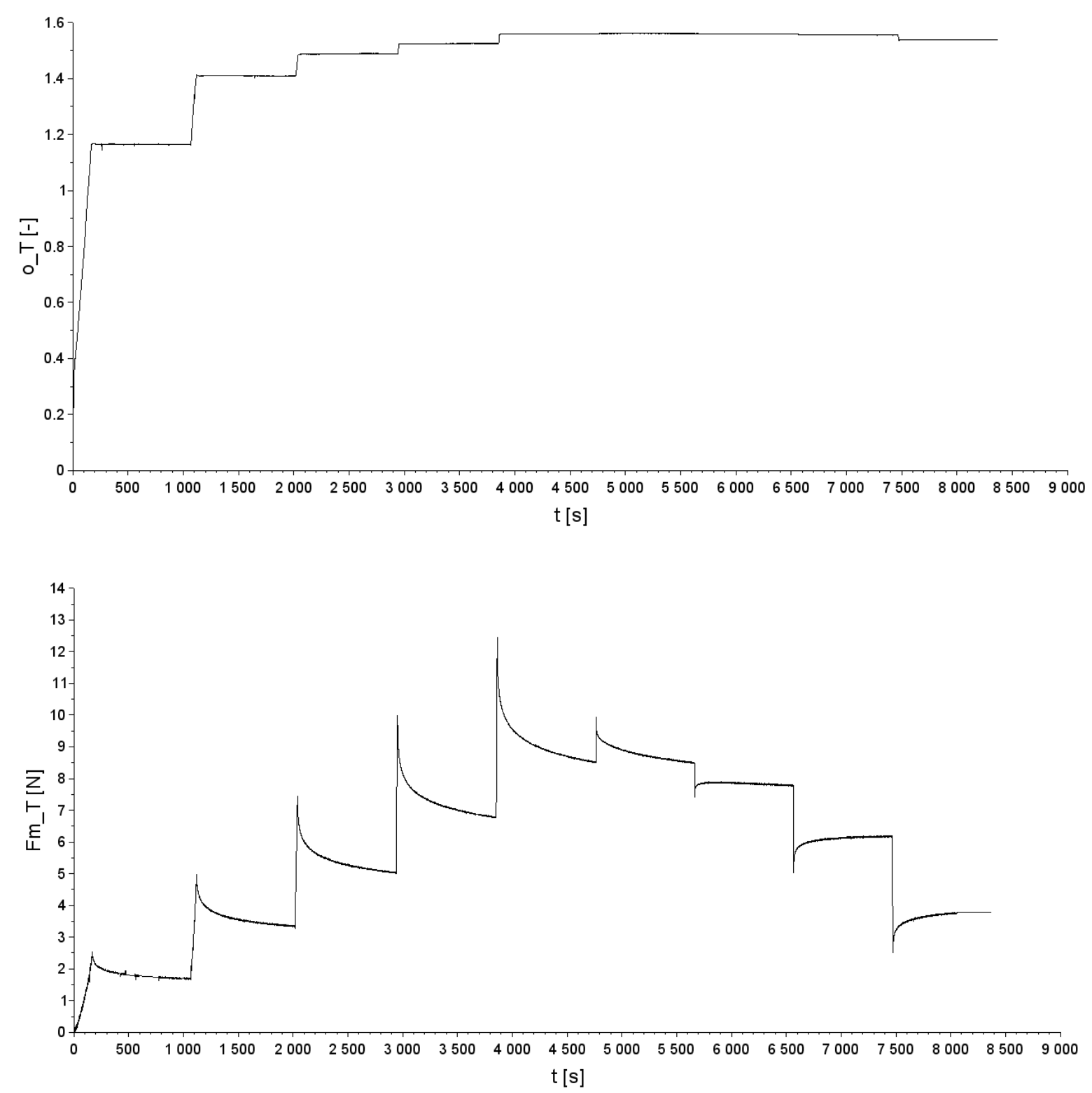

Figure 8: Result of a tensile test including relaxation stages of 15 minutes. Above: evolution of the opening in the T-direction of the mesh in the middle of the netting panel. Below: evolution of the force by mesh $F m_{T}$ applied on the mesh in the middle of the panel.

\subsection{Suspending test}

During suspending tests, different steps were performed: netting panels were submitted to forces added at the bottom, one after another, of 1.3, 3.257, 7.18 and $11.1 \mathrm{~N}$. For each different level of solicitation, there was a creep period of $30 \mathrm{~min}$. Figure 11 shows the mean value $( \pm$ standard deviation) of the heights $L_{\text {panel }}$ measured on the 10 suspended netting samples. 


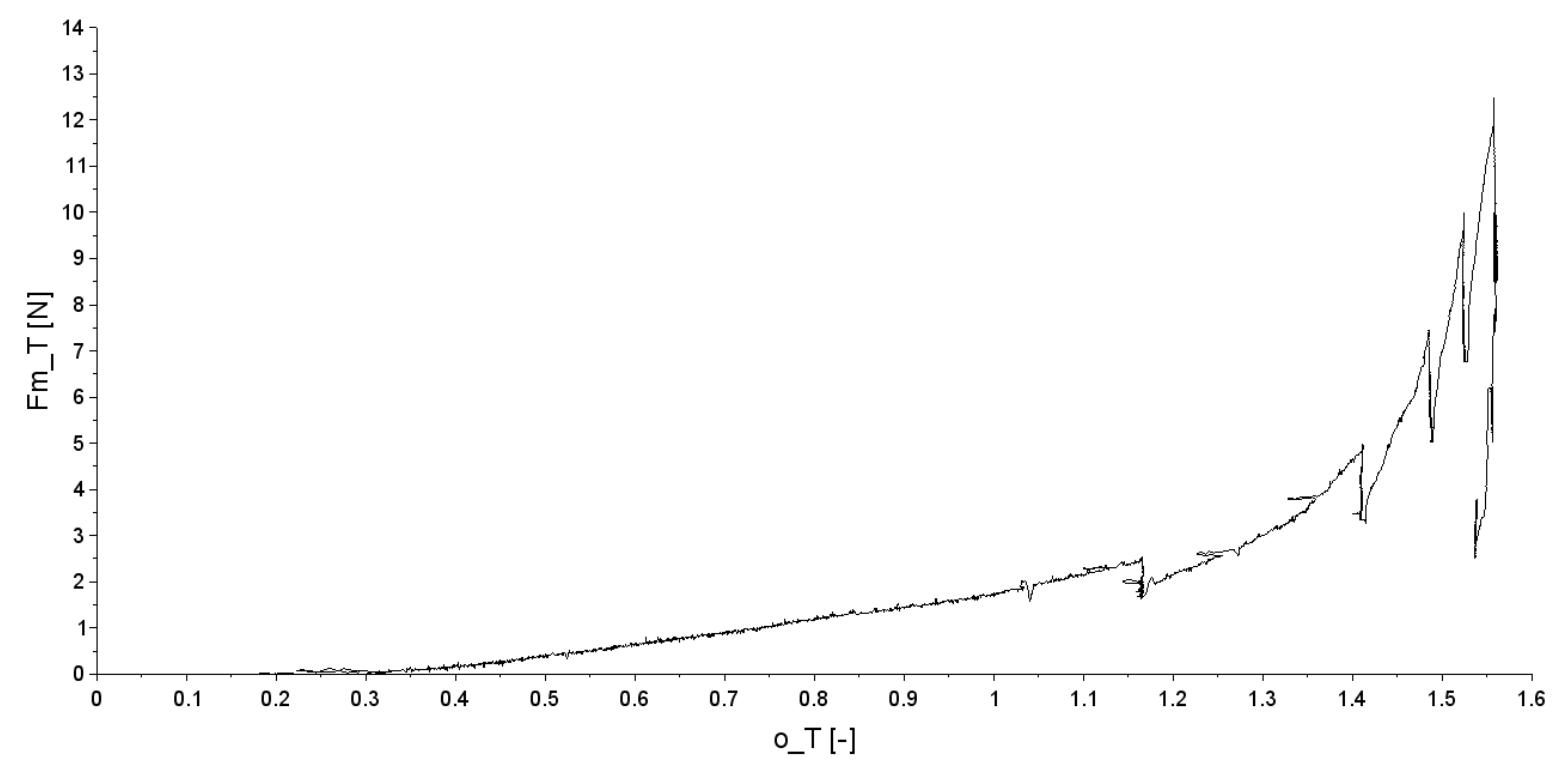

Figure 9: Result of a tensile test including relaxation stages.

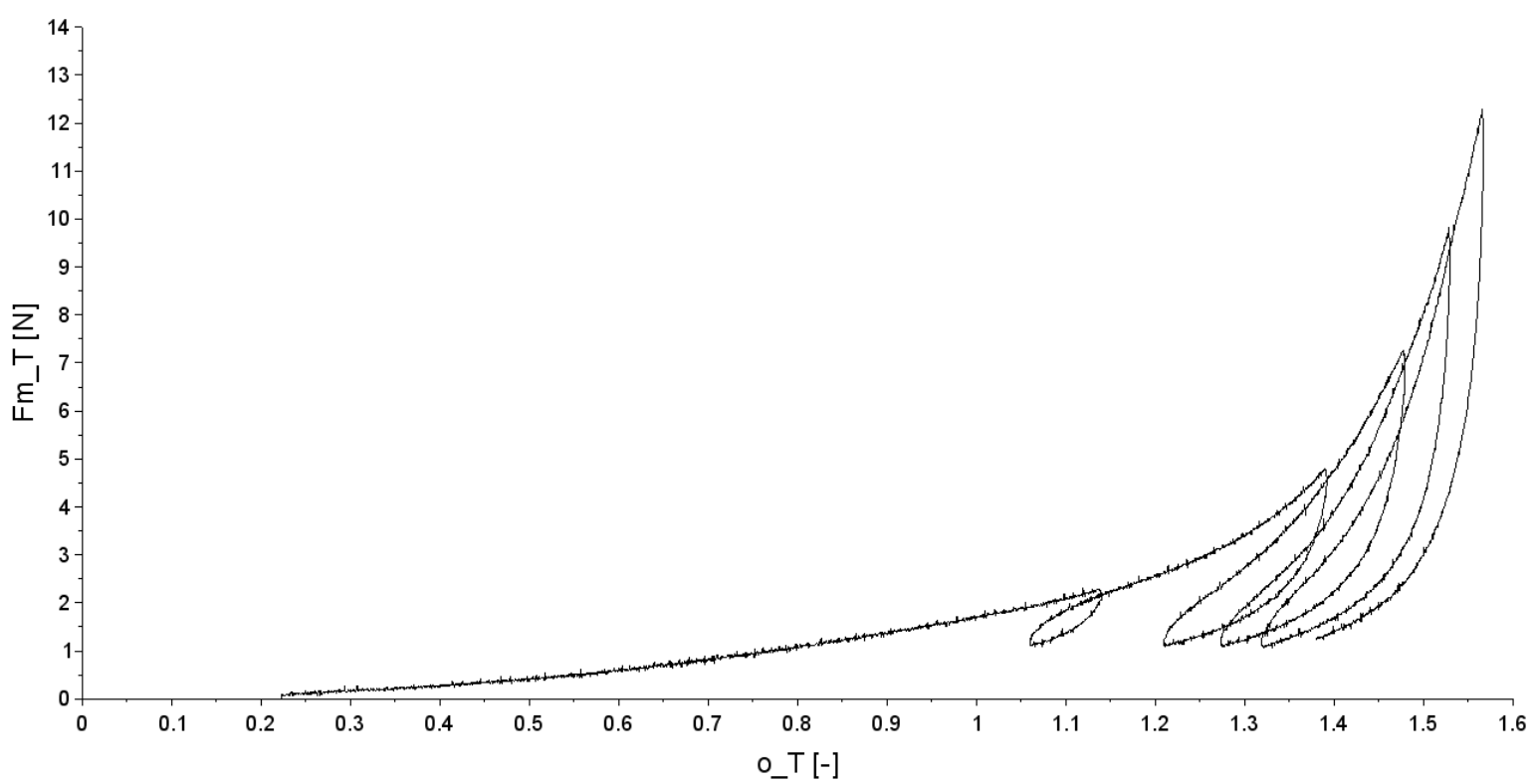

Figure 10: Result of a cyclic tensile test on a double twine netting sample.

\section{NUMERICAL METHODS}

\subsection{Analytical model}

An analytical model based on the beam theory and allowing large rotations is proposed to simulate the tensile test, assuming that the load applied on the mesh in the middle of the netting panel is supposed to be uniaxial, only in the T-direction. The notations used are defined in figure 12

First, the bending moment $M$ is related to the curvature as follows: 


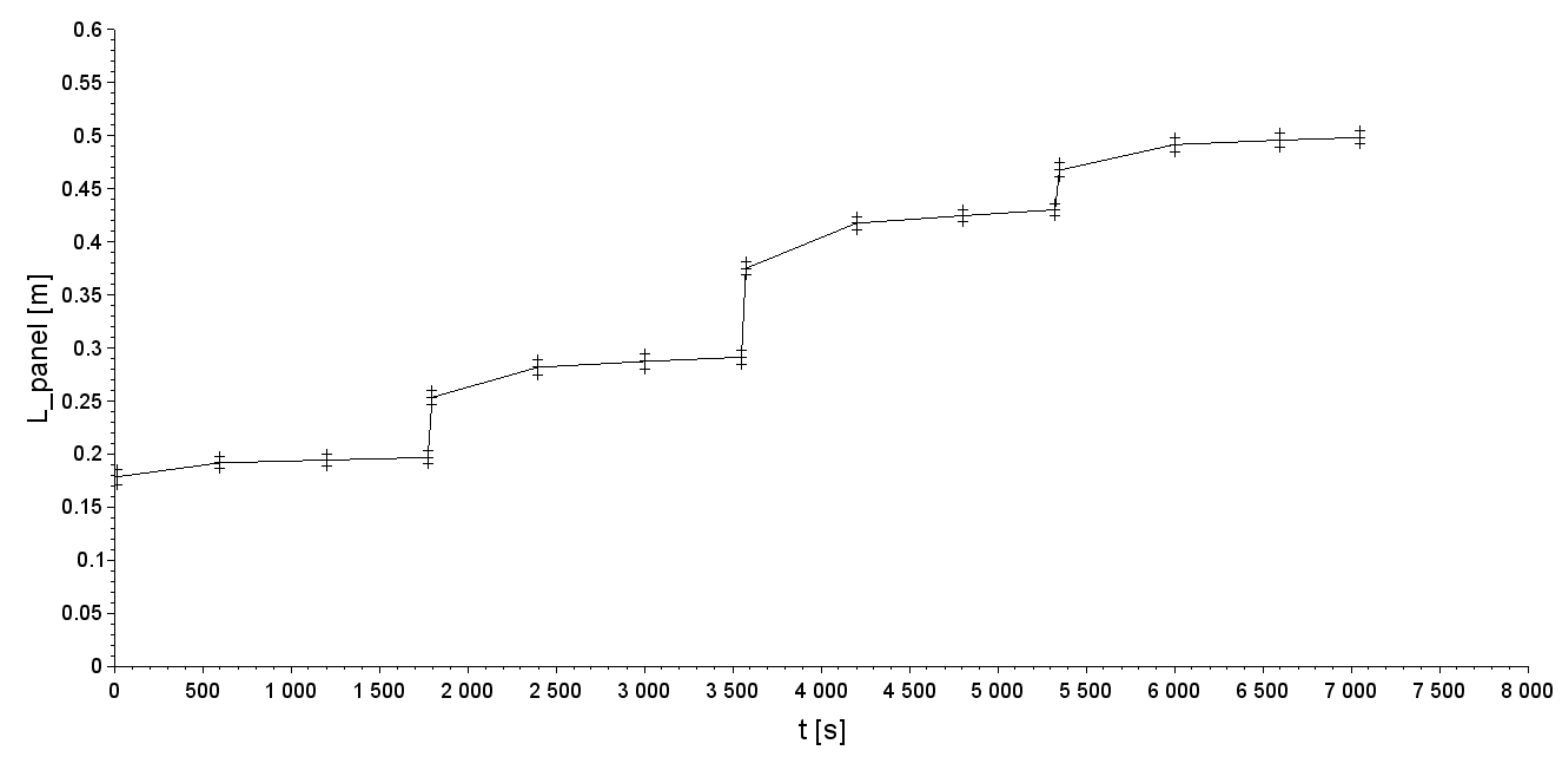

Figure 11: Evolution of the mean value ( \pm standard deviation) of the heights $L_{\text {panel }}$ measured on the 10 suspended netting samples.
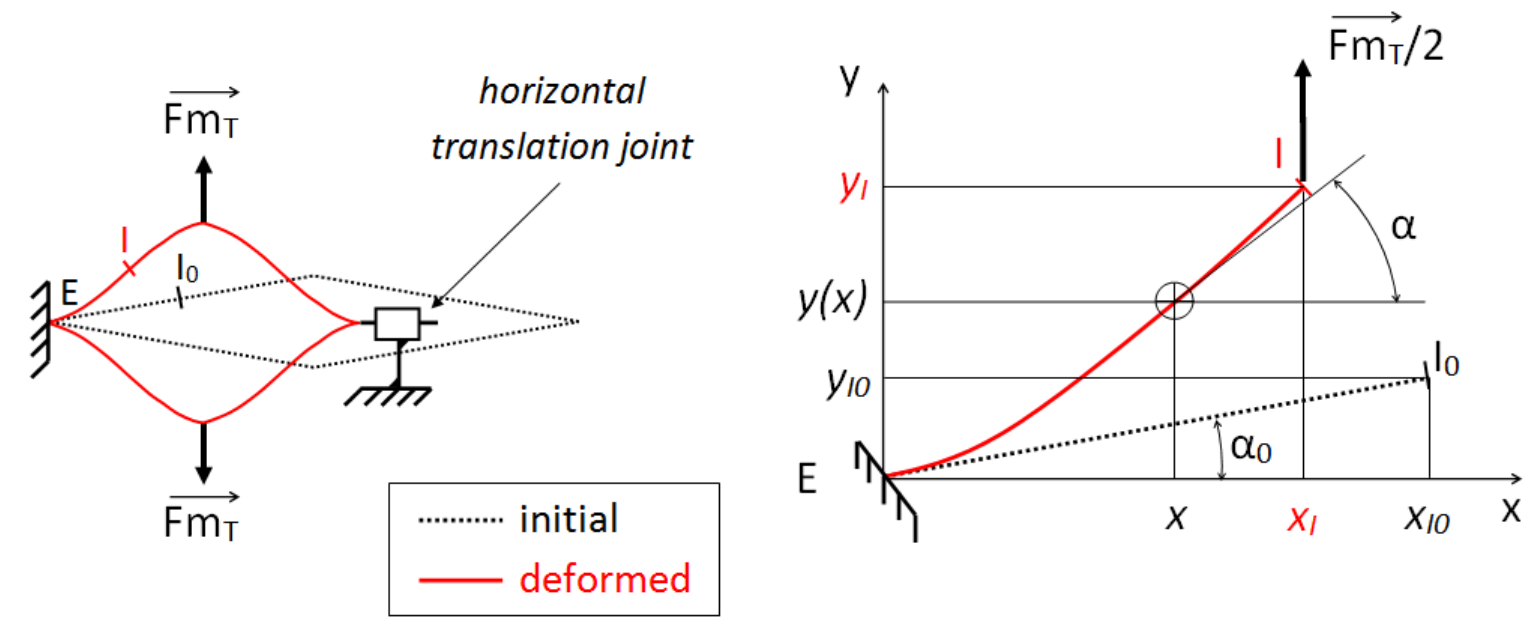

Figure 12: Left: application of a load $F m_{T}$ on a mesh. Right: kinematic of the twine between the points $E$ and $I$.

$$
M=E I \frac{d \alpha}{d s}
$$

where $E I$ is the bending stiffness, $s$ is the curvilinear abscissa, $\alpha$ is the mesh angle, and $\frac{d \alpha}{d s}$ is the curvature.

The bending moment is given by:

$$
M=\frac{F m_{T}}{2}\left(x_{I}-x\right)
$$


along the center line of the beam.

The curvature is a function of $y(x)$ as follows:

$$
\left(\frac{d \alpha}{d s}\right)=\cos ^{2}(\alpha) y^{\prime \prime}(x) \frac{1}{\sqrt{1+\left[y^{\prime}(x)\right]^{2}}}=\frac{y^{\prime \prime}(x)}{\left[1+\left[y^{\prime}(x)\right]^{2}\right]^{3 / 2}}
$$

By using relations 3, 4 and 5, the following expression is obtained:

$$
\forall x \in\left[0 ; x_{I}\right] \quad \frac{F m_{T}}{2}\left(x_{I}-x\right)=E I \frac{y^{\prime \prime}(x)}{\left[1+\left[y^{\prime}(x)\right]^{2}\right]^{3 / 2}}
$$

The boundary conditions are:

$$
\begin{gathered}
y(x=0)=0 \\
\alpha(x=0)=\alpha_{0} \Leftrightarrow y^{\prime}(x=0)=\tan \left(\alpha_{0}\right)
\end{gathered}
$$

The length of the mesh sides is assumed to be constant:

$$
\begin{gathered}
\forall t \in \mathbf{R}^{+} \quad L_{m s}=L_{m s 0} \\
\int_{E}^{I} \mathrm{~d} s=\int_{E_{0}}^{I_{0}} \mathrm{~d} s=\frac{L_{m s 0}}{2} \\
\frac{L_{m s 0}}{2}=\int_{0}^{x_{I}} \frac{\delta s}{\delta x} \mathrm{~d} x=\int_{0}^{x_{I}} \sqrt{1+\left[y^{\prime}(x)\right]^{2}} \mathrm{~d} x
\end{gathered}
$$

To work with dimensionless parameters, $\bar{x}$ and $\bar{y}$ are defined by:

$$
\bar{x}=\frac{2 x}{L_{m s 0}} \quad \bar{y}=\frac{2 y}{L_{m s 0}}
$$

And $u$ is defined by:

$$
\forall x \in\left[0 ; x_{I}\right] \quad u(\bar{x})=y^{\prime}(x)
$$

Using equations 12 and 13 , relations 6,8 and 11 become 14,15 and 16 respectively.

$$
\forall \bar{x} \in\left[0 ; \bar{x}_{I}\right] \quad \frac{F m_{T}}{2}\left(\frac{L_{m s 0}}{2}\right)^{2}\left(\bar{x}_{I}-\bar{x}\right)=E I \frac{u^{\prime}(\bar{x})}{\left[1+[u(\bar{x})]^{2}\right]^{3 / 2}}
$$




$$
\begin{gathered}
u(\bar{x}=0)=\tan \left(\alpha_{0}\right) \\
\int_{0}^{\bar{x}_{I}} \sqrt{1+[u(\bar{x})]^{2}} \mathrm{~d} \bar{x}=1
\end{gathered}
$$

The solutions of the differential equation 14 are:

$$
\forall \bar{x} \in\left[0 ; \bar{x}_{I}\right] \quad \frac{F m_{T}}{2 E I} \frac{L_{m s 0}^{2}}{4} \bar{x}\left[\bar{x}_{I}-\frac{\bar{x}}{2}\right]=\frac{u(\bar{x})}{\sqrt{1+[u(\bar{x})]^{2}}}+K
$$

where $K \in \mathbf{R}$.

Using relation 15 with relation 17 , we obtain:

$$
\forall \bar{x} \in\left[0 ; \bar{x}_{I}\right] \quad \frac{F m_{T}}{2 E I} \frac{L_{m s 0}{ }^{2}}{4} \bar{x}\left[\bar{x}_{I}-\frac{\bar{x}}{2}\right]=\frac{u(\bar{x})}{\sqrt{1+[u(\bar{x})]^{2}}}-\frac{\tan \left(\alpha_{0}\right)}{\sqrt{1+\tan ^{2}\left(\alpha_{0}\right)}}
$$

To simplify the equation, we defined $v$ by:

$$
\forall \bar{x} \in\left[0 ; \bar{x}_{I}\right] \quad v(\bar{x})=\frac{u(\bar{x})}{\sqrt{1+[u(\bar{x})]^{2}}}
$$

So $u$ can also be defined by:

$$
\forall \bar{x} \in\left[0 ; \bar{x}_{I}\right] \quad u(\bar{x})=\frac{v(\bar{x})}{\sqrt{1-[v(\bar{x})]^{2}}}
$$

where $v \in]-1 ; 1[$.

Then relation 18 leads to:

$$
\forall \bar{x} \in\left[0 ; \bar{x}_{I}\right] \quad v(\bar{x})=\frac{F m_{T}}{2 E I} \frac{L_{m s 0}^{2}}{4} \bar{x}\left[\bar{x}_{I}-\frac{\bar{x}}{2}\right]+\sin \left(\alpha_{0}\right)
$$

By using relations 20, 21 and 16, the value of $\bar{x}_{I}$ can be evaluated by an iterative algorithm (e.g. dichotomy algorithm).

Using the relations 12 and $13, \bar{y}$ becomes:

$$
\forall \bar{x} \in\left[0 ; \bar{x}_{I}\right] \quad \bar{y}(\bar{x})=\int_{0}^{\bar{x}} u(\bar{x}) \mathrm{d} \bar{x}
$$

So $\bar{y}_{I}$ can be calculated by:

$$
\bar{y}_{I}=\int_{0}^{\bar{x}_{I}} u(\bar{x}) \mathrm{d} \bar{x}
$$

Finally, the displacement of the half mesh side, and so the opening of the mesh in the middle of the netting panel during the tensile test, submitted to a tensile force $F m_{T}$, can be calculated by relations 20,21 and 23 . 


\subsection{Finite element model}

The mechanical behaviour of the studied netting was modeled using the Abaqus Standard finite element code. A mesh side was assumed to behave like a Timoshenko beam. In Abaqus, we model a mesh side with a planar beam that uses linear interpolation and a $2 \mathrm{D}$ hybrid formulation (B21H type in Abaqus). Timoshenko beams allow for transverse shear deformation and can be subject to large axial strains. Hybrid beam element types are used for geometrically nonlinear analysis when the beam undergoes large rotations. In this study, we modeled a mesh side with $20 \mathrm{~B} 21 \mathrm{H}$ elements. The shear modulus was supposed to be very high so that the transverse shear deformation was not taken into account. The axial stiffness $E A$ of the beam elements was determined using the results presented in figure 5 .

\subsection{Inverse identification methods}

The global scheme for the identification of one mechanical parameter, here the bending stiffness, is presented on Fig. 13 .

In case of the tensile test, the objective function was the difference between the experimental and the numerical height of the mesh in the middle of the netting panel. In case of the suspending tests, the objective function was calculated by using two different methods: first, the objective function was the mean distance between the coordinates of the experimental and the numerical knots; then, the objective function was the difference between the experimental and the simulated suspended sample height. There was a difference of 3.5 per cent between the bending stiffness identified using the measured coordinates of all the knots and the bending stiffness evaluated using the height of the netting panel. Thus, it was decided to identify the parameter with only the height of the netting sample.

The optimization of the search of the parameter EI is performed with the Nelder-Mead (or downhill simplex) algorithm proposed by John Nelder and Roger Mead ([8]).

This algorithm is used within the Python library SciPy, which contains an optimization module. The convergence is usually reached after about ten loop iterations of the Nelder-Mead algorithm.

\section{NUMERICAL RESULTS}

\subsection{Tensile test}

Using the experimental results and the identification method presented previously, we identified the bending stiffness $E I$ of braided twines constituting the netting sample. The evolution of the bending stiffness $E I$ is presented on figure 14 . The evolutions of this parameter during the whole test, including relaxation stages, identified with the finite element model and the analytical model, are presented.

Figures 15 and 16 show, respectively, the influence of the force by mesh in the T-direction and the influence of the opening $o_{T}$ on the bending stiffness. For these two figures, the bending stiffness was identified using the finite element model at the begining ( $0 \mathrm{~min})$ and at the end (15 $\min$ ) of the relaxation stages.

\subsection{Suspending tests}

The identified evolution of the bending stiffness, in the case of suspending tests, is presented on figure 17. As previously described, the identifications of the bending stiffness have been carried out using results of suspending tests on 10 netting samples. 


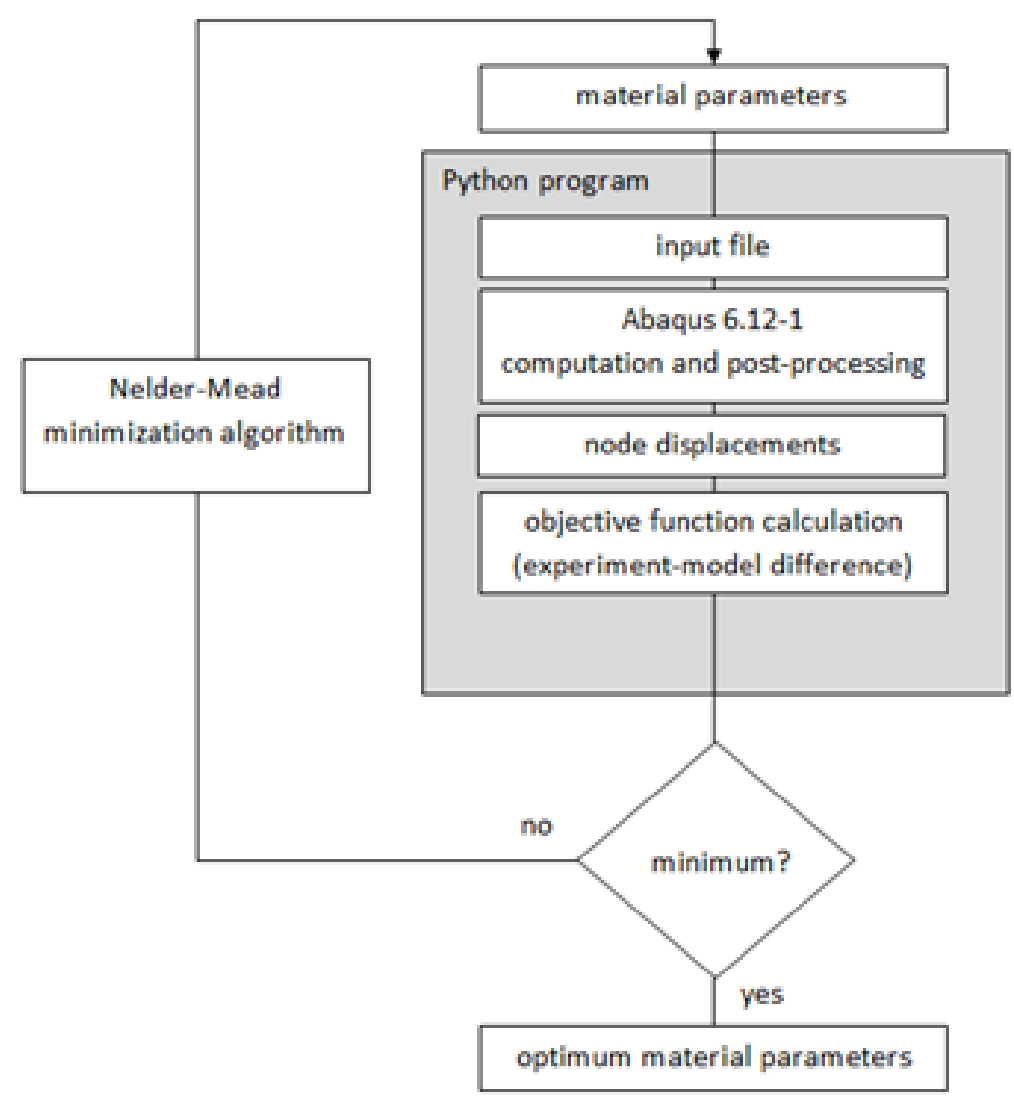

Figure 13: Inverse analysis strategy.

\section{DISCUSSION}

The tensile test provides results which reveal the viscosity of the material (Fig. 8 and 9). Figure 14 shows the importance of taking into account the viscosity of the material, which influences greatly the identified bending stiffness. Thus, with the assumption of a viscoelastic behaviour, the relaxation stages permit to reduce the effect of viscosity and to improve the evaluation of the bending stiffness; indeed, the higher the relaxation duration is, the less the rate $d E I / d t$ is, then the less the effect of the time on the value of $E I$ is. Figure 16 shows that the opening $o_{T}$ was still high during the unload (with relaxation stages of 15 minutes). So the mesh did not come back to its initial state. The structure presented a permanent deformation or a long-term viscosity.

Figure 14 shows that the bending stiffness identified with the analytical model is lower than the one evaluated with the finite element model. The relative difference between the two curves ranges from $4.40 \%$ for a force by mesh value of $1.59 \mathrm{~N}$ to $12.77 \%$ for a force by mesh value of $12.38 \mathrm{~N}$. According to the finite element model of tensile test, the mesh in the middle is submitted to a tensile force in the $\mathrm{T}$-direction and a compression force in the $\mathrm{N}$-direction. However, the analytical model only takes into account the tensile force. We can assume that to obtain the same mesh opening in T-directon without compression force in the $\mathrm{N}$-direction, a lower bending stiffness is necessary. We can note that for a low force level, in case of a tensile test, the bending stiffness identified with the analytical model is close to the one evaluated with the 


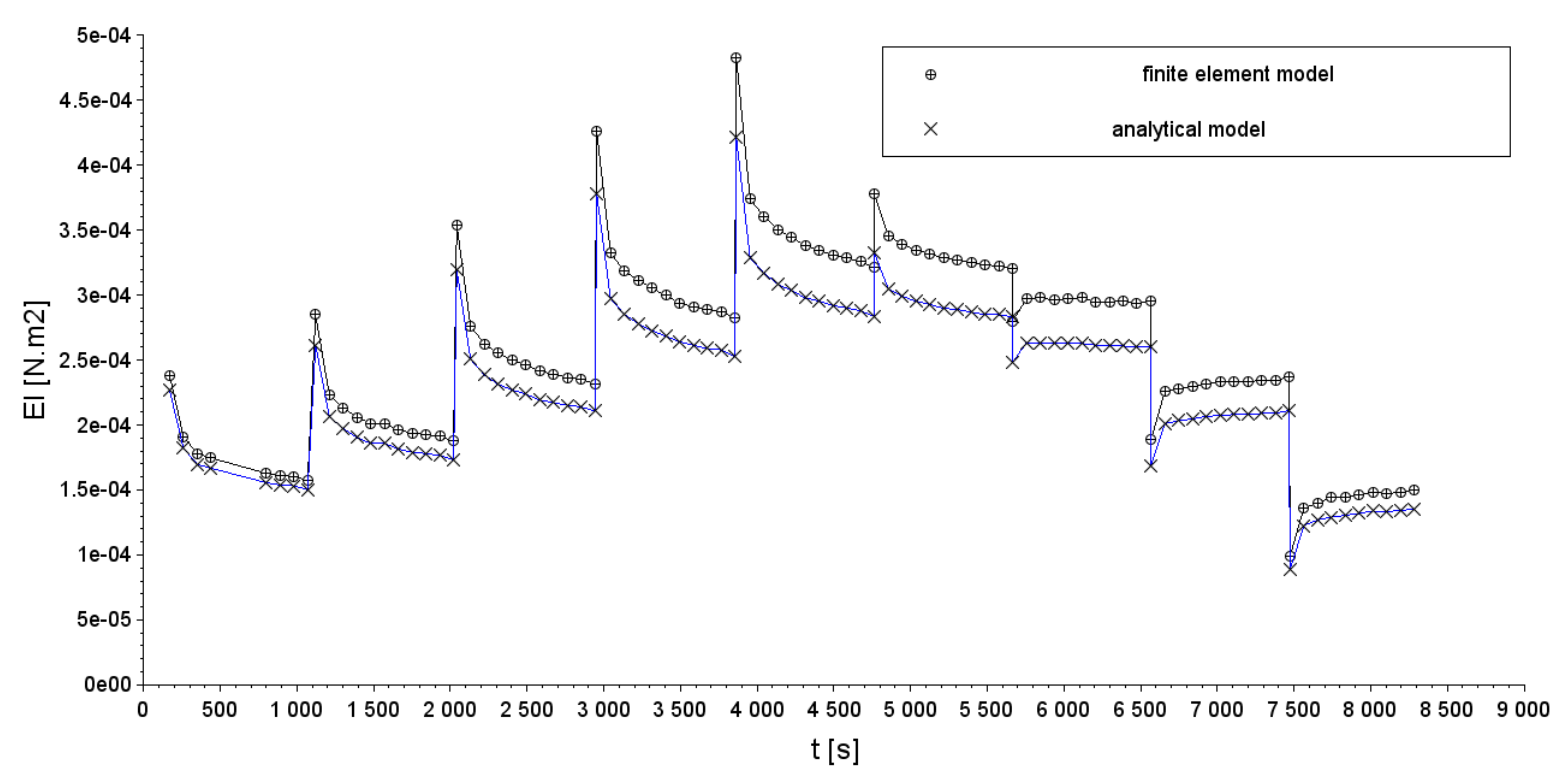

Figure 14: Tensile test. Evolution of the bending stiffness $E I$, identified with the finite element model (' $\oplus$ ', black line) and the analytical model (' $x$ ', blue line).

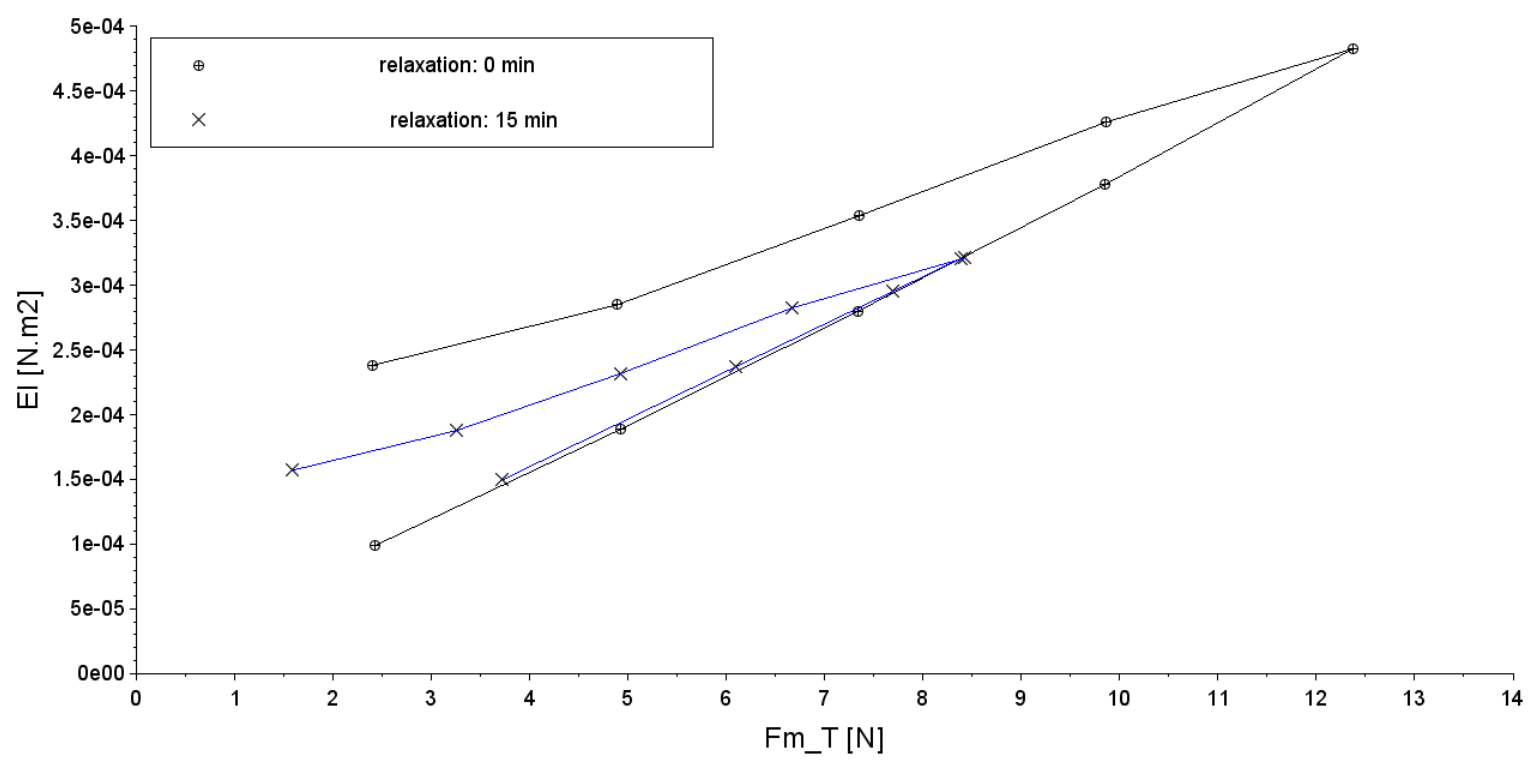

Figure 15: Tensile test. Evolution of the bending stiffness $E I$, identified using the finite element model at 0 minute (' $\oplus$ ', black line) and at 15 minutes (' $\times$ ', blue line) of each relaxation stage, as a function of the applied load $F m_{T}$.

finite element model.

Figures 15 and 16 show the evolution of the identified bending stiffness as a function of the force applied on the mesh $\mathrm{Fm}_{T}$ and as a function of the opening $o_{T}$ respectively. We can note that the variation of the parameter $E I$ during the test is smaller when it has been calculated at the end of each relaxation step. However, the evolution of $F m_{T}$ (Fig. 8 ) indicates that the contribution of the viscosity is not completely relaxed after a relaxation stage of 15 minutes.

When the opening increases (and so the force), the tensile force in mesh sides increases so 


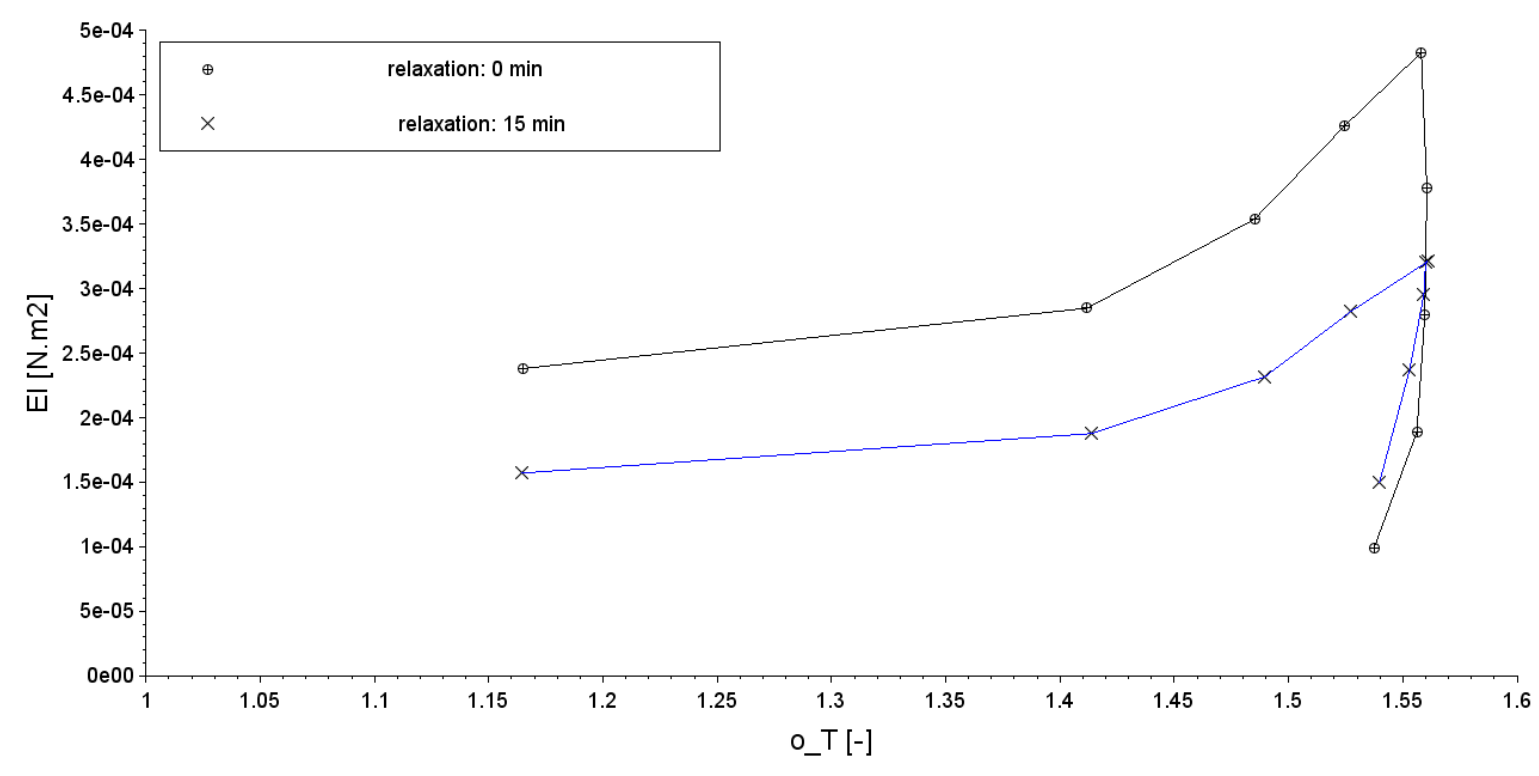

Figure 16: Tensile test. Evolution of the bending stiffness $E I$, identified using the finite element model at 0 minute (' $\oplus$ ', black line) and at 15 minutes (' $\times$ ', blue line) of each relaxation stage, as a function of the opening in the T-direction, $o_{T}$.

we could suspect that the twine diameter decreases. And a smaller diameter means a smaller moment of inertia $I$. Thus, we could suspect a decrease of $E I$ when the opening increases. But figure 16 shows that $E I$ increases when the opening $o_{T}$ of the mesh increases. It is necessary to take into account the complexity of the twine structure. When the twine diameter decreases, interactions between fibres (directions) and interactions between the core and the sheath become different. The complexity of the behaviour was already revealed on figure 5 which showed that it was not possible to identify a constant Young modulus $E$.

During the tensile test, the force by mesh at the beginning of relaxation stages ranged from $2.40 N$ to $12.38 N$. During suspending tests, netting panels were submitted to forces by mesh added at the bottom ranging from $0.3235 \mathrm{~N}$ to $2.775 \mathrm{~N}$. The force by mesh applied on the uppermost meshes was higher and ranged from $0.46 \mathrm{~N}$ to $2.91 \mathrm{~N}$ due to the effect of gravity. The load applied on meshes during suspending tests were only around a quarter of the load applied during the tensile test. With this loading level, a smaller opening $o_{T}$ of the mesh is observed during the suspending test than during the tensile test, therefore a smaller variation of the identified bending as presented on figure 17 . We can note that the bending stiffness identified for the smallest value of force by mesh with the tensile test is close to the ones identified with the suspending tests.

The suspending tests on 10 netting samples allow us to calculate the first and third quartiles, and the standard deviation of the bending stiffness (Fig. 17). The standard deviation of the bending stiffness decreases when the load increases, so when the opening of meshes increases. We can remember that each sample was subject to a pretension step (loaded in tensile with $392.4 \mathrm{~N}$ in the $\mathrm{N}$-direction during 30 minutes). Thus, we can suppose that the decrease of the standard deviation is not caused by the tightening of knots. 

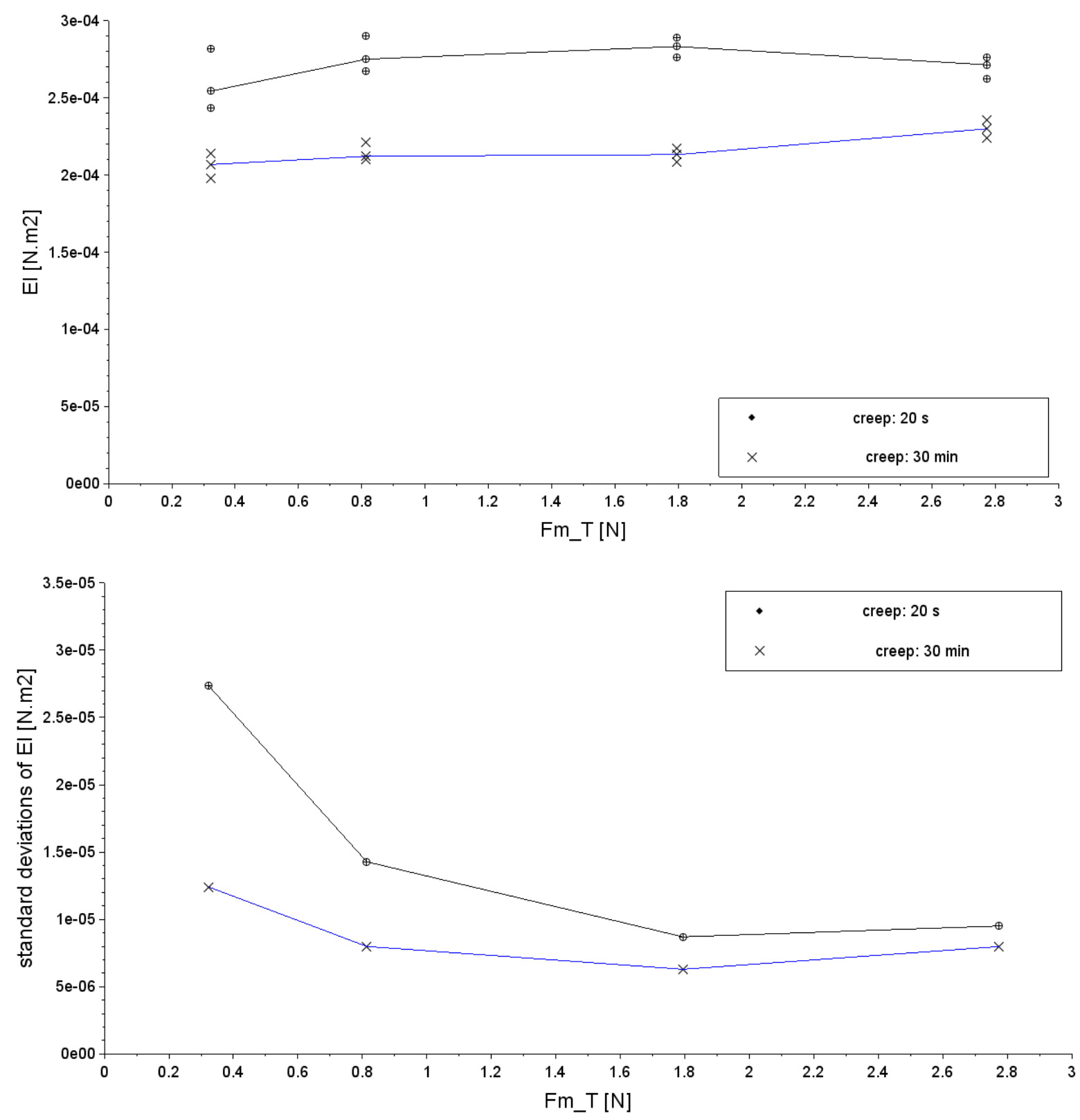

Figure 17: Suspending test. Medians of the bending stiffness EI with first and third quartiles (above) and standard deviation (below) of EI at the begining (20 s) and at the end (30 min) of each creep stage.

\section{CONCLUSION}

A simple method to evaluate the bending stiffness of braided twines, so the mesh opening stiffness of fishing nets, is presented. In this study, twines were made up of polyethylene.

Two different tests were performed: a tensile test and a suspending test. In case of a tensile test, a load and unload cycle interrupted by several relaxation stages was imposed. In case of suspending test, netting panels were submitted to forces added at the bottom, with creep stages. The experimental results show the importance of taking into account the viscosity of the material.

Two models based on the beam assumption were developped: an analytical model and a finite 
element model. The results obtained with these models are close, even if the bending stiffness identified with the analytical model, in case of the tensile test, is lower than the bending stiffness evaluated with the finite element model. The finite element model is more accurate because it models the compression force in the $\mathrm{N}$-direction.

The results of the identifications show an evolution of the bending stiffness as a function of time (viscosity), of the load level and of the mesh opening. To take into account the effect of the structure of the braided twine, one could model the fibres constituting the core and the sheath of each twine, and the interactions between the fibres. This work provides experimental results that could be used to study the contact-friction interactions, to develop a model for large deformation analysis of fibrous material.

Finally, the identification of the bending stiffness EI on suspending test, proposed here, could be used as a simple methodology to evaluate the mesh opening stiffness of fishing nets.

\section{ACKNOWLEDGMENT}

This research received the support of Region Bretagne under a financing agreement for doctoral research (no. 13/2.213 747/F).

\section{REFERENCES}

[1] P. Badel, E. Vidal-Sall, P. Boisse, Large deformation analysis of fibrous materials using rate constitutive equations. Computers and Structures, 86, 1164-1175, 2008.

[2] G. Bles, W.K. Nowacki, A. Tourabi, Experimental study of the cyclic visco-elasto-plastic behaviour of a polyamide fibre strap. International Journal of Solids and Structures, 46, 2693-2705, 2009.

[3] M. K. Broadhurst, D. J. Sterling, R. B. Millar, Increasing lateral mesh openings in penaeid trawls to improve selection and reduce drag. Fisheries Research, 170, 68-75, 2015.

[4] D. Durville, Contact-friction modelling within elastic beam assemblies: an application to knot tightening. Comput Mach, 49, 687-707, 2012.

[5] B. Herrmann, F.G. O'Neill, Theoretical study of the influence of twine thickness on haddock selectivity in diamond mesh cod-ends. Fisheries research, 80, 221-229, 2006.

[6] ISO, Fishing nets - Netting — Basic terms and definitions. International Standard Organisation, 1107, ISO Geneva, 1974.

[7] W. Moderhak, Influence of twine parameters on the shapes of meshes and T90 codends. International Workshop Methods for the Development and Evaluation of Maritime Technologies(DEMaT'07), ROSTOCK, Germany, 2007.

[8] J.A. Nelder, R. Mead, A Simplex Method for Function Minimization. The Computer Journal, 7, 308-313, 1965.

[9] F.G. O'Neill, Bending of twines and fibres under tension. Journal of the Textile Institute, 93, 1-8, 2002.

[10] F.G. O'Neill, A theoretical study of the factors which influence the measurement of fishing netting mesh size. Ocean engineering, 30, 2053-2063, 2003. 
[11] A. de la Prada, M. Gonzales, Nonlinear stiffness models of a net twine to describe mesh resistance to opening of flexible net structures. J Engineering for the Maritime Environment, 2014.

[12] A. de la Prada, M. Gonzales, Quantifying mesh resistance to opening of netting panels: experimental method, regression models, and parameter estimation strategies. ICES Journal of Marine Science, 2014.

[13] D. Priour, A finite element method for netting: application to fish cages and fishing gears. Springer, 2013.

[14] D. Priour, J.-Y. Cognard, Investigation of methods for the assessment of the flexural stiffness of netting panels. 10th International Workshop Methods for the Development and Evaluation of Maritime Technologies (DEMaT'11), SPLIT, Croatie, 2011.

[15] J.H.B. Robertson, P.A.M. Stewart, A comparison of size selection of haddock and whiting by square and diamond mesh codends. J. Cons. Int. Explor. Mer, 44, 148-161, 1988.

[16] A. Sala, F.G. O’Neill, G. Buglioni, A. Lucchetti, V. Palumbo, RJ. Fryer, Experimental method for quantifying resistance to the opening of netting panels. ICES Journal of Marine Science: Journal du Conseil, 64, 1573-1578, 2007. 